\title{
The Processing Method and Application of Medical Image with Feature Points Matching
}

\author{
Tian Zhongyong \\ Zhengzhou people's Hospital ,Blood purification Department,Zhenghou 450053,China
}

Keywords: feature points matching; medical images; registration; correlation coefficient

\begin{abstract}
In this thesis, the registration method of medical image is based on feature points, and mainly starting from the feature extraction operator. It makes comparative studies on the aspects of accuracy, stability and so on. Through theoretical and experimental studies, we make improvements on algorithm. Feature points matching in this thesis regards feature point neighborhood gray scale correlation as the similarity measure, uses affine transformation of space to search space, and utilizes affine invariance of Mahalanobis distance correcting feature point matching so as to the optimization of parameters. Experimental results show that the improvement method of extraction operator based on Harris in this thesis makes a good effect on some certain kind of medical images.
\end{abstract}

\section{Introduction}

The rapid development of medical imaging technology and the sharp growth of image data play a huge promoting role in the field of medical image processing and analysis. In order to assist doctors in making diagnosis, operation planning, assessment of treatment effects and so on, the way how to quantify the extraction and analyze the structure, function and pathological information in image has gradually become a focus of attention in the field of medical image analysis. Medical image segmentation and registration just are the basis and premise of the research hotspots. While, because of the existence of many sorts of noise as well as artifacts interference in medical images, and the diversity and individual difference of all the tissues and lesions, there are lots of problems existing in medical image segmentation and registration as well. The doctors deliver related commands to the assistant mechanical arms through the computer, and directly manipulate the handle, button and other main hard equipment on the control console; through supervising assistants' operation conditions, and adjusting as well as refining, they aim to achieve expected results; finally they complete the minimally invasive operation. Operation mechanical arms are operated by contacting with patients directly. Therefore, its performance indicators scale will directly influence the performance, effect and safety performance of the whole operation robot system. On the other hand, the doctors live understand the condition of patient operation site through two HD camera on the operation arms, so they are directly influenced on the judge of patients' situations by the clarity and accuracy of transmitted image which are also important research trends of operation navigation system. Thus, the further study of image processing technology of robots' binocular stereo vision in minimally invasive surgical operation has significant theoretical and practical application value in clinical medicine.

The thesis studies and applies SIFT operator. Thorough the optimization of the algorithm, SIFT operator becomes very fast and can be completed in all kinds of real-time processing applications. The basic theory of SIFT operator is realized by Gauss Pyramid hierarchical form, and the extraction of feature descriptors from feature points at all scales contributes to the stability of the algorithm. The determination of feature points' direction requires to comprehensively evaluate the local information statistics in main direction and auxiliary direction, which further improves the accuracy.

\section{Image registration algorithm based on feature}

Registration algorithm based on feature extracts the image feature (Harris Registration, Canny Registration, some regional characteristics of contour and so on). To Establish space transformation 
model through the relationship among features can largely reduce the amount of data on computers. Through using all sorts of features extracted from target image, we overcome the influence of rectum, bladder and other tissue on registration and achieve good results. Fig. 2-6 is local feature through partial extraction. The $\mathrm{L} I$ indicates image along the $\mathrm{X}$ direction of first-order partial derivative, and LiLi indicates image along the $\mathrm{X}$ direction of second-order partial derivative, so it is with other operations. The method to combine image local feature and image gray registration method is feasible. Correctly introducing feature into registration method based on gray scale can positively influence space deformation field, and modify the image position which optimization algorithm can't process due to large deformation, so as to make the results of image registration more accurate.

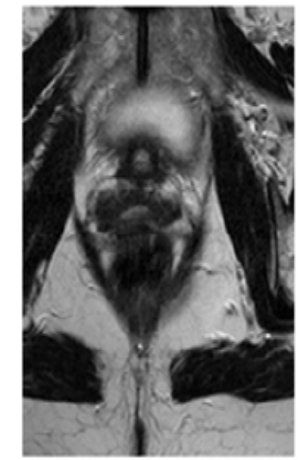

(a)

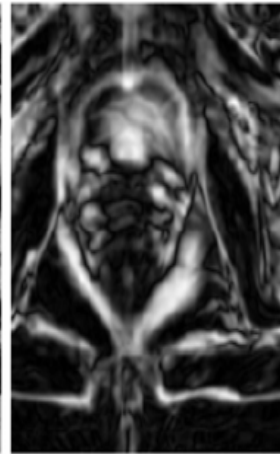

(b)

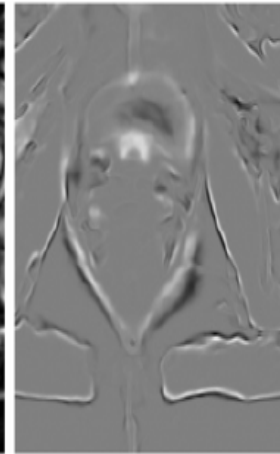

(c)

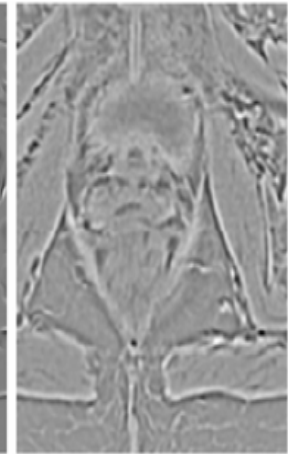

(d)

Fig.1 (a) is actual image, (b) is $L_{i} L_{i}$ image feature of operation extraction, (c) $L_{i} L_{i j} L_{j}$ image feature of operation extraction, (d) $\mathrm{L}_{\mathrm{ii}}$ image feature of operation extraction.

\section{Medical image registration based on corner feature}

\subsection{Corner extraction}

In all kinds of features, feature points is a stable and effective feature, which is invariant to rotation and can overcome gray scale inversion. Applying feature point to matching process can not only reduce the amount of computation involved without losing important gray scale information of image, but also can make similar matching for similar parts of image. While the method of image registration based on corner just avoids the defect mentioned above. It focuses on the variance of gray scale in pixel neighborhood rather than the edge contour of the whole target. As a result, using corner method can complete a better image registration effect. The common operator of feature points extraction includes Moravec Operator, Forstner Operator, Harris Operator, SUSAN Operator, SIFT Operator and so on. Harris algorithm and SUSAN algorithm are two most widely applied algorithm. This paper will carry out vital research on these two algorithm.

Design a local detection window in the image. When the window performs tiny movement along each direction, we have to detect the average energy change of the window. Furthermore, when the energy change value exceeds a set threshold, the central pixel of the window will be extracted as corner, marked as pixel $(x, y)$ whose gray scale is $f(x, y)$. Each pixel $(x, y)$ of the image moves causes gray scale $(\mathrm{u}, \mathrm{v})$ intensity change, which is expressed as:

$$
E_{u v}(x, y)=\sum W_{u v}[f(x+u, y+v)-f(x, y)]^{2}
$$

In this formula, pixel movement $(\mathrm{u}, \mathrm{v})$ values $(1,0),(1, \mathrm{l}),(0, \mathrm{l}),(-1,0),(-1,-\mathrm{l}),(0$, -1) , ( , , -l) , apparently, Moravee algorithm can only detect the intensity change of window function moved in eight basic directions. It can not accurately extract all corner, and it is kind of sensitive to isolated pixel, noise and edge. Corner response function defined in algorithm is $\mathrm{C}(\mathrm{x}, \mathrm{y})=\mathrm{min}$ ( Euv $(\mathrm{x}, \mathrm{y}))$, so that it does not possess rotation invariance. 
Through differential operation and auto correlation matrix, detecting corner point differential operator can reflect the intensity change of gray scale pixel in arbitrary direction, so that it can effectively distinguish corner and edge and inhibit noise to a certain degree.

$$
\begin{aligned}
& E_{u v}(x, y)=\sum W_{u v}[f(x+u, y+v)-f(x, y)]^{2} \\
& =\sum_{u v}\left[x X+y Y+o\left(x^{2}+y^{2}\right)\right]^{2}
\end{aligned}
$$

Wuv is the coefficient of Gauss window (u, v), $\mathrm{X}=\partial f / \partial x, \mathrm{Y}=\partial f / \partial y$.

On the basis of feature analysis, enlightened by H.Morave's work, Harris and Stephens transformation the matrix form to be $M(I)$, herein, $M=M(I), 1 \in_{R 2}$, $M$ is defined as :

$$
M=(\nabla f)(\nabla f)^{T}=\left[\begin{array}{l}
f_{x} \\
f_{y}
\end{array}\right]\left[\begin{array}{ll}
f_{x} & f_{y}
\end{array}\right]=\left[\begin{array}{ll}
f_{x}^{2} & f_{x} f_{y} \\
f_{x} f_{y} & f_{y}^{2}
\end{array}\right]
$$

If $|\nabla \mathrm{f}|=0, \quad \mathrm{M}$ has characteristic value $\lambda 1=0$ and $\lambda 2=|\nabla \mathrm{f}| 2$. And the corresponding characteristic vectors is non-zero, which is the multiple of ( $-f x, f y)$ and ( $-f x$, fy ) $T$.

The matrix $M$ can analytically express edge features and point features. Suppose $G \geq 0$ to be a weighting function of $\iint G d x d y=1$, it can maintain the quality of original image. Thus, we can get:

$$
\bar{M}=G * M
$$

On the edge of ideal, the gradient of all points is on the same direction. Therefore, one characteristic value is $\mathrm{Q}$, and the other one is positive and large. On the edge of the truth, there is one small and one big characteristic value. As for the ideal feature point, the gradients of point is on orthogonal direction. Thus, there will be two same characteristic value, which are both big and almost in the same numerical size. In order to extract feature points, we make this expression:

$$
R_{\text {Harris }}=\operatorname{det} \bar{M}-k(\operatorname{tr} \bar{M})^{2}
$$

The $\operatorname{det} \bar{M}$ is the determinant (det $\bar{M}=\lambda_{1} \lambda_{2}$ ) of matrix M; $\operatorname{tr} \bar{M}$ is the trace $\operatorname{tr} \bar{M}=\lambda_{1}+\lambda_{2}$ of matrix $\mathrm{M}$; $\mathrm{k}$ is a parameters which is above zero, and it is recommended to take $\mathrm{K}=004$. $\operatorname{det} \bar{M}$ is small at the edge point and big at the characteristic point. $\operatorname{tr} \bar{M}$ keeps the same numerical size. Therefore, when RHarris value is the biggest in local position, it is feature point. Harris operator possesses better rotation and radiation invariance. It has better robustness to the influence of brightness variations of image, change of viewpoint and noise.

In order to avoid repeating the detection to choose suitable $\mathrm{k}$ value for many times, the thesis adopts corner response function to judge the corner position:

$$
R_{\text {Harris }}=\frac{\operatorname{det} \bar{M}}{\operatorname{Tr}(M)+\varepsilon}=\frac{A B-C^{2}}{A+B+\varepsilon}
$$

The improved Harris algorithm also adopts the histogram equalization and the adjacent point elimination strategy: choose a $3 \times 3$ template to process the whole image. Regarding the template as the center, we compare in its eight neighborhood. If there exists more than one corner in the template, we should only keep the largest corner of the $\mathrm{R}$ value, so as to achieve the aim of eliminating the neighboring point. The experimental results shows that this strategy can effectively inhibit the clustering phenomenon of corner. As shown in Fig.2, the improved Harris algorithm effectively avoids the clustering phenomenon of corner, so that the corner extraction is more evenly distributed in the whole image. 


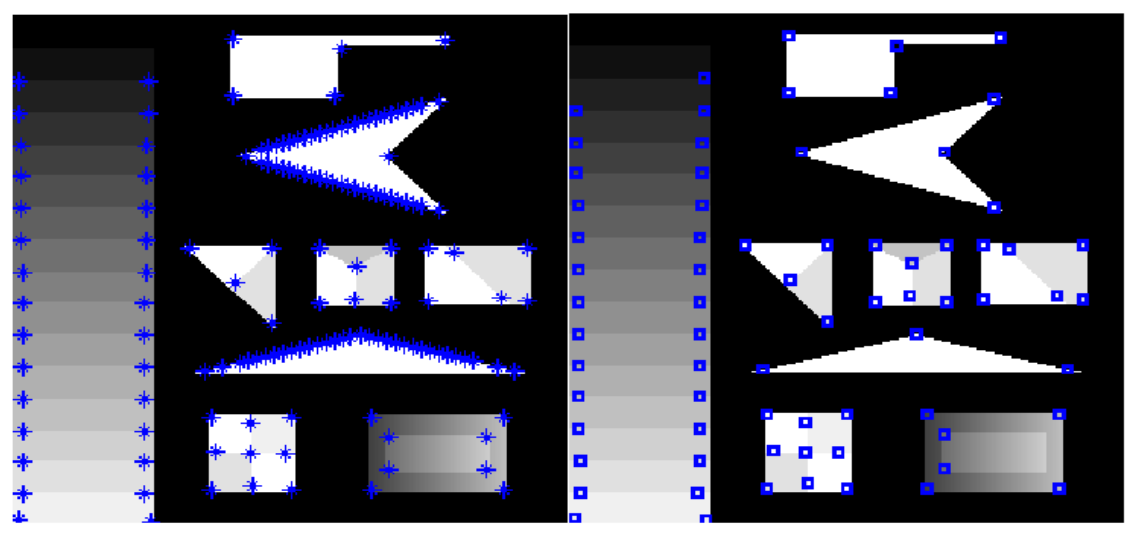

(a) is extraction result of Harris before improvement

(b) is extraction result of Harris after improvement

Fig. 2 is the extraction result of Harris before and after improvement

\subsection{Matching algorithm of feature points}

Establish a correspondence relationship of feature points by using the correlation of neighborhood gray scale of feature points and match the extracted feature points. Suppose two images $\mathrm{I} 1$ and I2 which are expected to be matched, and extract two feature points N1 and N2 respectively, $\mathrm{X} 1(\mathrm{x} 1, \mathrm{y} 1$ )and $\mathrm{X} 2(\mathrm{x} 2, \mathrm{y} 2)$ are arbitrary feature points extracted from the two images. Choose the size of the window to be $W=(2 n+1) \times(2 n+1)$, so the normalized cross correlation coefficient of X1 and X2 are:

$$
\operatorname{Cor}\left(X_{1}, X_{2}\right)=\frac{\operatorname{cov}\left(X_{1}, X_{2}\right)}{\operatorname{std}\left(X_{1}\right) \times \operatorname{std}\left(X_{2}\right)}
$$

Std, cov represent the standard deviation and correlation function of the image. Their definitions are :

$$
\begin{gathered}
\operatorname{cov}\left(X_{1}, X_{2}\right)=\frac{\sum_{i=-n}^{n} \sum_{j=-n}^{n}\left[I_{1}\left(x_{1}+i, y_{1}+j\right)-M\left(X_{q}\right)\right]\left[I_{2}\left(x_{2}+i, y_{2}+j\right)-M\left(X_{2}\right)\right]}{W} \\
\operatorname{std}(X)=\sqrt{\frac{\sum_{i=-n}^{n} \sum_{j=-n}^{n}[I(x+i, y+j)-M(X)]^{2}}{W}}
\end{gathered}
$$

In the formula, with regarding $\mathrm{X}(\mathrm{x}, \mathrm{y})$ as the center, $\mathrm{M}(\mathrm{X})$ is the average value of pixel gray scale point in the related window of images I1 and I2. M (X) is :

$$
M(x)=\left[\sum_{i=-n}^{n} \sum_{j=-n}^{n}[I(x+i, y+j)] / W\right.
$$

Calculate the normalized cross correlation of each feature point, so as to get cross correlation matrix N1 $\times$ N2. Set a threshold value as theta. If the cross correlation of the two point are bigger than theta, then we can say the two point matching.

During the process of registration, it is a major problem to select the method of coordinate transformation between appropriate images in the registration technology. The thesis choose the affine transformation that meets the rigid plane transformation relations. Affine transformation can be defined as : if transformation $S$ meets: $R n \rightarrow R n, S(x)=T(x)+a$, and $T$ is non-singular linear transformation, and $\mathrm{a} \in_{\mathrm{Rn}}$, transformation $\mathrm{S}$ is called affine transformation. Affine transformation can be expressed by the following matrix: 


$$
\left[\begin{array}{lll}
x & y & 1
\end{array}\right]=\left[\begin{array}{lll}
w & \mathrm{z} & 1
\end{array}\right] T=\left[\begin{array}{lll}
w & \mathrm{z} & 1
\end{array}\right]\left[\begin{array}{ccc}
t_{11} & t_{12} & 0 \\
t_{21} & t_{22} & 0 \\
t_{31} & t_{32} & 1
\end{array}\right]
$$

The specific form of this transformation is decided by the $T$ element value. Suppose reference image and image that is expected registration are affine relationship, and I1(x1,y1) ,I2( $\mathrm{x} 2$, y2 ) respectively represent corresponding points of reference image and image that is expected registration, they should satisfy the relation:

$$
\left[\begin{array}{l}
x_{2} \\
y_{2}
\end{array}\right]=\left[\begin{array}{ll}
a_{11} & a_{12} \\
a_{21} & a_{22}
\end{array}\right]\left[\begin{array}{l}
x_{1} \\
y_{1}
\end{array}\right]+\left[\begin{array}{l}
b_{1} \\
b_{2}
\end{array}\right]
$$

The registration transformation relation is just the required vector $p=\left(a_{11}, a_{12}, b_{1}, a_{21}, a_{22}, b_{2}\right)$.

The thesis use affine invariance of Mahalanobis distance to delete the point symetry whose error distance is too large, so as to improve the accuracy of matching point symerty. Mahalanobis distance is a common distance measure method. For considering the size of pattern characteristic parameters and the correlation between characteristics, it is an effective method to calculate the similarity between two unknown samples. For the sample $X=\{[\mathrm{x} 1, \mathrm{y} 1] \mathrm{T}, \ldots,[\mathrm{xn}, \mathrm{yn}] \mathrm{T}\}$, the Mahalanobis distance from any point in this sample $\mathrm{X}=[\mathrm{xi}, \mathrm{yi}]$ to $\mathrm{u}=[\mathrm{ux}, \mathrm{uy}]$ is:

$$
\begin{gathered}
\gamma_{i}=\left[(x-u) C_{x}(x-u)^{T}\right]^{1 / 2} \\
u=\left[u_{x}, u_{y}\right]^{T}=\frac{1}{n}\left[\sum_{i=1}^{n} x_{i}, \sum_{i=1}^{n} y_{i}\right] \\
C_{x}=\frac{1}{n} \sum_{i=1}^{n}\left[x_{i}-u_{x}, y_{i}-u_{y}\right]\left[x_{i}-u_{x}, y_{i}-u_{y}\right]
\end{gathered}
$$

According to formula (12), we calculate the Mahalanobis distance of $\Gamma 1$ and $\Gamma 2$, and define the sum of covariance of the corresponding points on the Mahalanobis distance as :

$$
\sigma=\sum_{i=1}^{m}\left(\gamma_{1 i}-\gamma_{2 i}\right)^{2} / 4
$$

In terms of the affine invariance of Mahalanobis distance, it exists $\Gamma 1=\Gamma 2, \sigma=0$. In the actual application, we can say that: when a set point symerty $\sigma$ is within a scope, this set point symerty possesses affine invariance. We can use affine invariance of Mahalanobis distance on points that have already established matching relationship, and select correct matching point stmetry.

\section{Examples of verification based on feature points matching algorithm}

After certain transformation on an MRI image, we can get a new image. Comparing it with its original image through registration with feature point matching method mentioned above, we can get the experimental result as Fig.3. From the result, we can see that the extracted points with improved Harris corner extraction algorithm are stable and with great robustness; in the thesis, the accuracy and reliability of medical image registration method based on feature points have achieved the expected goal. 


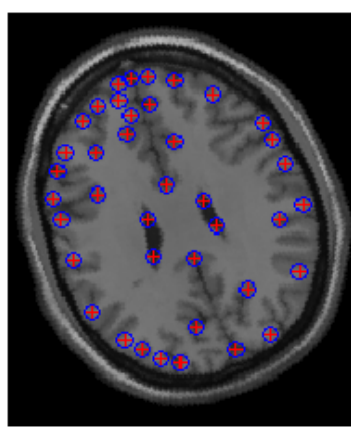

(a) image before corner extraction

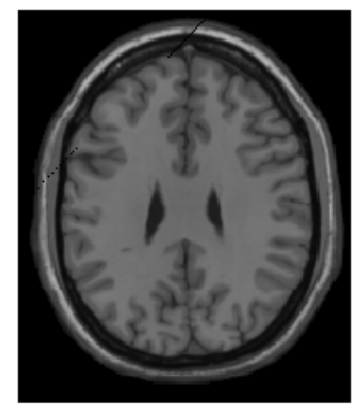

(c) image after registration

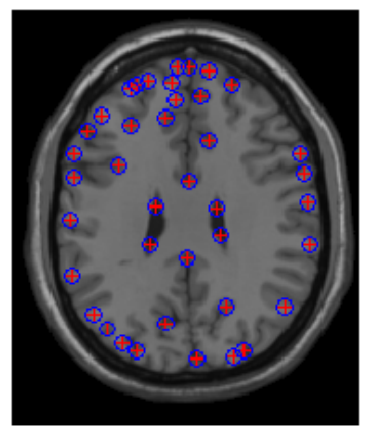

(b) reference image after corner extraction

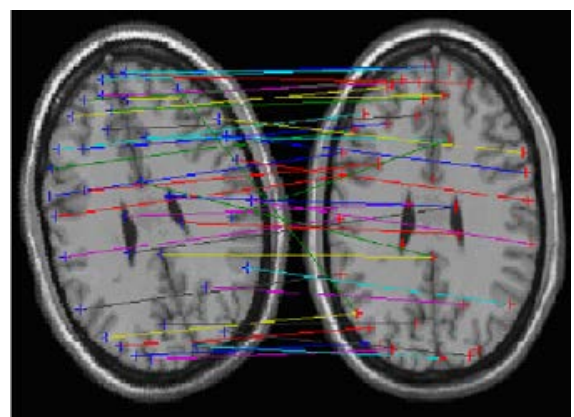

(d) corner matching image

Fig.3 results of experimental registration

\section{Conclusions}

In this paper, we use feature points neighborhood gray scale correlation to establish the correspondences of feature points, which is regarded as similarity measure, the affine transformation of space to search space, and affine transformation invariance of Mahalanobis distance to correct feature point matching, and finally, we use parameter refinement to improve the registration accuracy. It can be seen from the experimental results that the extracted points with improved Harris corner extraction algorithm are stable and with great robustness; in the thesis, the accuracy and reliability of medical image registration method based on feature points have achieved the expected goal.

\section{References}

[1] Dong Feng etc.. Automatic Image Registration Based on Generalized Feature Points matching[J]. Journal of Electronics and Information, 2005, 27(7):1013-1014.

[2] Xiao Qian, Lu Hongwei. Adaptive Corner Point Detection Based on Gauss[J]. Journal of Computer Aided Design and Graphics, 2003, 15(11):1358-1361.

[3] Luo Zhongyi, Liu Chengming. Fast Algorithm of Gray Scale Image Matching[J]. Journal of Computer Aided Design and Graphics, 2005, 17(151):966-969.

[4] Jie, Bao Shanglian, Zhou Mingquan. Medical Image Processing and Analysis[M]. Electronic Industry Press, 2003.

[5] Wang Dakai. Partial Differential Equation in Image Processing[M]. Science Press, 2008.

[6] Wen Tiexiang, Yang Feng. A New Curve Evolution Hybrid Model for Image Segmentation Algorithm[J]. Journal of Circuits and Systems, 2007, 12(4):48-52. 
[7] Ding Mingyue, Cai Chao.Medical Image Processing[M]. Higher Education Press, 2010.

[8] Leordeanu M, Zanfir A, Sminchisescu C. Semi-supervised Learning and Optimization forHypergraph Matching[A]. Proceedings of 2011 IEEE International Conference on ComputerVision [C], 2011, 2274-2281.

[9] Im K, Pienaar R, Lee J-M, Seong J-K, Choi YY, Lee KH, et al. Quantitative Comparison andAnalysis of Sulcal Patterns using Suical Graph Matching: A Twin Study [J]. Neurolmage,2011,57(3):1077-1086.

[10] Zheng Y, Lin S' Kang S,Xiao R, Gee J, Kambhamettu C. Single-Image VignettingCorrection from Gradient Distribution Symmetries[J]. IEEE Transactions on Pattern Analysis and Machine Intelligence, 2012, in press. 